\title{
Postoperative Delirium is a Risk Factor of Poor Evolution Three Years After Cardiac Surgery: An Observational Cohort Study
}

This article was published in the following Dove Press journal:

Clinical Interventions in Aging

\author{
François Labaste $\mathbb{I D}^{1,2}$ \\ Jean Porterie ${ }^{3}$ \\ Paul Bousquet ${ }^{\prime}$ \\ Bertrand Marcheix ${ }^{3}$ \\ Pascale Sanchez-Verlaan' \\ Bernard Frances ${ }^{4}$ \\ Philippe Valet ${ }^{2}$ \\ Cedric Dray ${ }^{2}$ \\ Vincent Minville (D) ${ }^{1,2}$

\begin{abstract}
'Anesthesiology and Intensive Care Department, CHU Toulouse, Toulouse, et Cardiovasculaires, INSERM UI 048, Université de Toulouse, Université Paul Sabatier, Toulouse, France; ${ }^{3}$ Cardiac Surgery Department, CHU Toulouse, Toulouse, France; ${ }^{4}$ Research Center on Animal Cognition, Center for Integrative Biology, Toulouse University, CNRS, UPS, Toulouse, France
\end{abstract} \\ France; ${ }^{2}$ Institut des Maladies Métaboliques
}

Background: After cardiac surgery, postoperative delirium (POD) is common and is associated with long-term changes in cognitive function. Impact on health-related quality of life (QOL) and long-term dependence are not well known. This aim of this study is to evaluate the role of POD in poor evolution at three years after surgery including poor QOL and dependence and mortality.

Patients and Methods: We enrolled and followed 173 patients 60 years of age or older who were planning to undergo cardiac surgery with cardiopulmonary bypass. The primary composite outcome was death of any causes, or patients with either a loss of QOL (evaluated with of EuroQuol verbal 5D EQ5D less than 50), or a loss of two points on the instrumental activities of daily living occurring three years after surgery. POD was diagnosed with the use of Confusion Assessment Method. Multivariate logistic regression was performed.

Results: At three years, 74 patients $(42.8 \%)$ had a poor evolution. Independent risk factors in poor patient evolution were sex (female gender; OR: 3.6; 95\%CI: $1.45-8.7 ; p=0.006$ ), metabolic status (diabetic patients; OR: 4; 95\%CI: 1.6-10.2; $p=0.002$ ), Euroscore 2 (Euroscore $2>1.5$; OR: 5.2; 95\%CI: 1.7-15.4; $p=0.003$ ) and POD (OR: $3.3 ; 95 \% \mathrm{CI}$ $1.4-7.8 ; p=0.006)$. Coronary disease was protective (OR: $0.3 ; 95 \% \mathrm{CI}: 0.14-0.71 ; p=0.006$ ). Conclusion: After cardiac surgery, POD significantly altered patient evolution and increased risk of dependence and loss of QOL.

Keywords: cardiac surgery, delirium, dependence, quality of life, mortality

\section{Introduction}

Postoperative delirium (POD) and postoperative cognitive dysfunction (POCD) are of particular concern in older patients who have undergone cardiac surgery with use of cardiopulmonary bypass (CPB). ${ }^{1}$ Despite the declining incidence of complications following cardiac surgery, the reported incidence of POCD has remained largely unchanged, occurring in 30 to $65 \%$ of patients at hospital discharge. ${ }^{2-4}$ Thus, POD (both postoperatively and in the ICU) must be actively screened. ${ }^{5}$ Both POD and POCD delirium have been linked to serious negative consequences, including postoperative cognitive dysfunction, prolonged mechanical ventilation, and prolonged hospital and ICU stay, as well as increased health care costs and long-term cardiovascular events after cardiac surgery. ${ }^{6}$ Age and degree of inflammatory response after CPB explains the high incidence of the diseases in this population. ${ }^{7-9}$ Indeed age, low ejection fraction, diabetes, and extracardiac arteriopathy were found to be independent predictors of postcardiac surgery delirium in
Correspondence: Vincent Minville Department of Anesthesia and Intensive Care Medicine, Toulouse University

Hospital, Toulouse, France

Tel +33561322791

Email minville.v@chu-toulouse.fr 
elderly patients. Postoperative risk factors of developing delirium for patients above 65 years were atrial fibrillation, postoperative pneumonia, elevated postoperative creatinine, and prolonged hospital LOS. ${ }^{6,10}$ Recently, preoperative administration of dexamethasone has reduced the inflammatory response and thereby decreased the risk of early POCD after cardiac surgery. ${ }^{11}$ The surgery aim is not only to treat the cardiac diseases but also to maintain or to improve functional capacities and QOL (quality of life) and to avoid loss of autonomy. However, there is little data in the literature. In fact, there are few studies on the medium-term and long-term consequences of POCD and POD on patients' QOL and autonomy. ${ }^{12,13}$ In addition, with regard to heart surgery, cognitive changes are still mainly studied in the framework of coronary artery bypass graft. ${ }^{12}$

The main objective of this study was to evaluate the role of POD in poor evolution at three years after cardiac surgery including poor QOL and dependence and mortality. Then, we researched factors, which explicated a poor patient evolution, especially influence of postoperative cognitive trajectories.

\section{Patients and Methods}

We conducted a prospective, monocentric longitudinal study in a cardiovascular surgery department of the Toulouse University Hospital. This work was approved by the local research ethics committee (Comité d'éthique pour la recherché du CHU de Toulouse no. 78-1014) in 2015. According to French legislation, given the observational nature of this analysis, the ethics committee specifically approved verbal informed consent, and the consent was both verbal and informed. Consent was orally obtained from the study participants prior to study commencement. Patients were included between November 2014 and May 2015 and were followed at three years after the surgery, between November 2017 and May 2018.

All patients were 60 years of age or older and undergoing planned cardiac surgery with use of CBP, either coronary artery bypass grafting, heart valve surgery, or both. The exclusion criteria included all patients who refused to participate, patients who were unable to complete the questionnaire by telephone (language barrier, deafness, etc), patients under protective measures, patients who had undergone heart surgery without use of $\mathrm{CPB}$ and patients less than 60 years of age.

Anesthesia was induced with sufentanil, propofol and cisatracurium and maintained with sevoflurane, sufentanil and cisatracurium. During and after CPB, sevoflurane was stopped and propofol $1 \%$ was administered in a targetcontrolled infusion adapted to a bispectral index monitoring system (BIS, Medtronic, MI, USA). BIS index monitoring was initiated before the induction and was maintained at 40-60 throughout the procedure.

Norepinephrine was used as a first-line vasopressor in case of postoperative hypotension. The combination of norepinephrine and dobutamine was considered in case of postoperative right or left or both ventricular failure. If necessary, epinephrine was administered in second-line.

Just after surgery, patients were admitted in an intensive care unit (ICU) where they were awake and monitored for at least the first $24 \mathrm{~h}$.

Clinical and biological data related to the health history, preoperative elements and the ICU and standard health care unit data were collected.

The presence of POD was verified on a daily basis. Trained research personnel, including nurses, assessed POD. The Confusion Assessment Method (CAM) adapted for the ICU (CAM-ICU) was used when the patient was admitted in our ICU. ${ }^{1}$ The CAM was used when the patient was transferred to the ward. The main criteria were researched: sudden onset, inattention, disorganized thoughts, alteration in level of consciousness, and associated criteria (disorientation, memory loss, abnormal perception, psychomotor disorders and sleepwake disorder). If any of these indicators were present, the patient was scored as delirious. Patients were assessed on the first seven days after surgery, three assessments were daily performed. The duration of delirium was calculated as number of days between the initial positive delirium assessment and the final positive delirium assessment.

Cognitive function was assessed one day before surgery (baseline) by medical trained personnel. We chose the MMSE (Mini Mental State Examination) as the measure of cognitive function because it is commonly used in geriatric patients and was used for POCD diagnostic. ${ }^{7}$ After surgery, MMSE was performed on days one and seven. Patients were classified with POCD on discharge when they lost two points or more between baseline and day seven.

At three years, patients' evolution was investigated. A phone call with the patient or family was performed. The callers were blinded to the patients' cognitive pathway. Mortality, QOL and autonomy were assessed. Quality of life data were assessed gathered with the EQ5D-3L tool. 
This is a self-evaluation tool with five dimensions (mobility, self-care, activities of daily living, pain/discomfort and anxiety/depression). It also includes a visual analog scale (VAS) that reflects the feelings of patients concerning their overall health status, which we adapted into a numerical scale between 0 and 100 to ensure its completion. This instrument, through which 243 health statuses can be defined, is usually used in other studies for phone interviews, notably in cardiology. The EQ5D-3L scale is also recommended by the HAS (French High Health Authority) and by the National Institute for Health and Care Excellence (NICE) for health care "cost-utility" analyses. The degree of autonomy for the activities of daily living was assessed with an instrumental activity scale IADL (Instrumental Activities of Daily Living). It is used to assess autonomy through a total score ranging from 0 to 5 for men and 0 to 8 for women. This tool is recommended by the HAS and INSERM (French Institute for Health and Medical Research) for example, to assess cognitive deterioration in current activities of daily life (functional assessment).

The primary study outcome was a composite outcome measure which described poor patient evolution. It was death of any cause, or patients with either a loss of QOL (evaluated with of EuroQuol verbal 5D EQ5D less than 50 ), or a loss of two points on the IADL occurring three years after surgery. Secondary outcome measures were the independent evaluation of quality of life, autonomy and mortality according to the occurrence or non-occurrence of postoperative delirium and POCD on discharge. In addition, we assessed the influence of POD on POCD on discharge from hospital. Finally, we attempted to identify the risk factors of delirium in our population.

\section{Statistical Analysis}

The study was carried out using MedCalc ${ }^{\circledR}$ statistical software version 15 (Mariakerke, Belgium). After the first descriptive statistics phase and verification of the distribution of values (Kolmogorov-Smirnov test), the studied population was separated into two groups according to the presence or absence of significant change in outcome. The characteristics of the patients in the two different groups were compared using nonparametric tests (Mann-Whitney test) for continuous variables. The results were expressed in median and $95 \% \mathrm{CI}$. The categorical variables for the two groups were compared with Fisher's exact test. We evaluated the association between the different covariates and the variable explained (significant change in outcome) in a multivariate analysis (logistic regression) by the odds ratio measurement. We used a stepwise regression model (backward elimination), which included all the variables with $p<0.05$ and then progressively removing the nonsignificant variables.

\section{Results}

Between November 2014 and May 2015, 197 patients were included. In the second phase, between November 2017 and May 2018, of the 197 patients monitored, 24 were lost to follow-up and excluded from the analysis. A total of 173 patients were included in the analysis. The characteristics of the patients are indicated in Supplementary Materials. In this population, $61(35.3 \%)$ patients presented a postoperative delirium and 63 patients $(36.4 \%)$ developed a POCD on discharge.

At three years after surgery, 74 patients $(42.8 \%)$ presented a poor evolution. The distribution within the main composite outcome measure was the following: 30 patients $(17.3 \%)$ had died, 18 patients $(10.4 \%)$ had a loss of autonomy with a decrease by more than two points on the IADL scale, 35 patients (20.2\%) had an EQ5D NRS less than or equal to 50 out of 100 . Moreover, in the group of patients with a significant alteration in the quality of life, the median NRS was 50.5 compared to 79.7 in the control group.

The results of our univariate analysis are presented in supplementary Table S1. We noted a median age of 69 years in the control group compared with 78 years in the group with poor evolution $(p<0.001)$, MMSE ( 25 vs 26 ; $p<0.001)$ and the presence of delirium with a duration more than or equal to one day $(52.7 \%$ vs $22.2 p<0.001)$. In addition, the duration of delirium was significantly longer in the group with poor evolution in comparison to the control group. The decrease in MMSE during hospitalization was greater in the group with poor evolution in comparison to the control group (two points vs one point, $p=0.01)$. In addition, POCD on discharge from hospital was more frequent in the poor evolution patient group. The intubation period and the ICU period were similar in the two groups. The CBP duration was also similar (72 min vs 79 min, $p=0.42$ ).

The results of our multivariate analysis are presented in Table 1. The independent risk factors for poor evolution in patient outcome were, sex (female gender; OR: 3.6; 95\% CI: $1.45-8.7 ; p=0.006$ ), metabolic status (diabetic patients; OR: $4 ; \quad 95 \% \mathrm{CI}: 1.6-10.2 ; \quad p=0.002)$, Euroscore 2 (Euroscore $2>1.5$; OR: 5.2; 95\%CI: 1.7-15.4. $p=0.003$ ) and POD (OR: $3.3 ; 95 \% \mathrm{CI}: 1.4-7.8 ; p=0.006)$. In addition, 
Table I Risk Factors for a Significant Alteration in Outcome: Multivariate Analysis

\begin{tabular}{|l|l|l|l|l|}
\hline & P & OR & OR Lower Level (95\%) & OR Upper Level (95\%) \\
\hline Gender (female) & 0.006 & 3.6 & 1.45 & 8.7 \\
Ischemic heart disease & 0.006 & 0.3 & 0.14 & 0.71 \\
Diabetes & 0.003 & 4 & 1.6 & 10.2 \\
Euroscore 2 >1.5 & 0.003 & 5.2 & 1.7 & 15.4 \\
Delirium & 0.006 & 3.3 & 1.4 & 7.8 \\
Age >69 years & 0.2 & 2 & 0.7 & 5.8 \\
SAPS2 >27 & 0.2 & 1.9 & 0.8 & 4.9 \\
\hline
\end{tabular}

Notes: Occurrence of significant alteration in outcome was modeled as a function of all predictors that differed $(p<0.10)$ in the univariate analyses. Multivariate logistic regression analysis was performed by using a backward (conditional) stepwise procedure. AUC of this model was 0.86 , the Hosmer-Lemeshow test was 0.85 ; $80.4 \%$ of patients were high-ranked by this model.

Abbreviations: SAPS II, Simplified Acute Physiology Score 2; AUC, area under the curve.

the presence of coronary heart disease was a protective factor in terms of significant alteration in the outcome in three years (OR: $0.3 ; 95 \% \mathrm{CI}: 0.14-0.71 ; p=0.006$ ). Finally, age and SAPS2 (Simplified Acute Physiology Score) did not appear to be significant $(p=0.2)$. The other variables that were tested provided no significant information, especially POCD on discharge from hospital that did not represent an independent risk factor for a poor patient evolution. Therefore, preoperative MMSE and the duration of delirium were not significantly associated with the occurrence of the main outcome measure.

The changes in MMSE score according to the presence or absence of delirium are shown in supplementary Table S2. In the group of patients without delirium, the preoperative MMSE was higher than in the group of patients with delirium. In addition, in the group of patients with delirium,

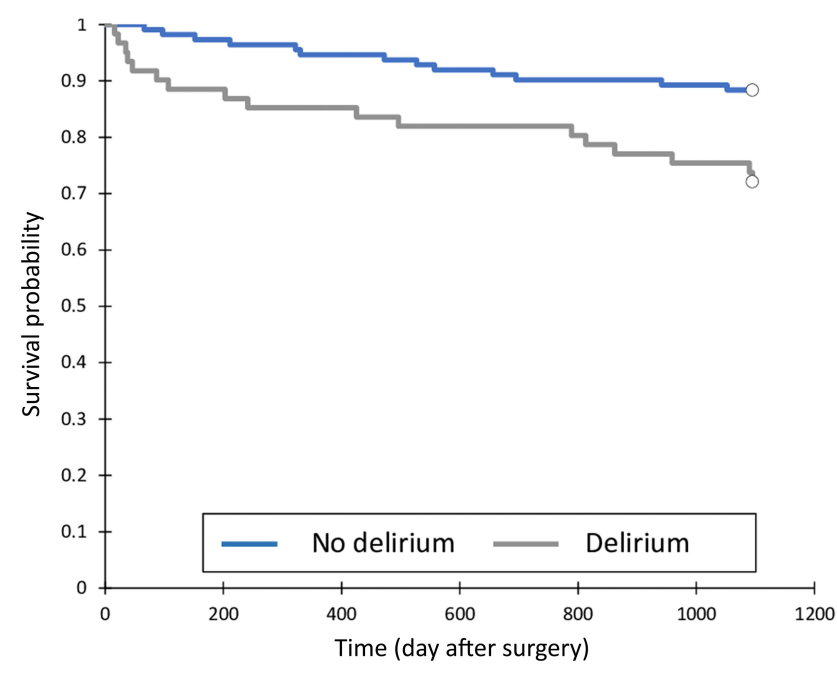

Figure I Kaplan-Meier time-to-event curves show the cumulative incidence of death from any cause among patients who present or not a postoperative delirium. At three years after the surgery, $56.7 \%$ of the postoperative delirium patients vs $30.8 \%$ in the control group (Wilcoxon test: $p=0.005$ ).

Abbreviation: POD, Postoperative delirium. a significant decrease in the preoperative and postoperative D7 MMSE score was noted. In the group without delirium, the D7 postoperative MMSE was similar to the preoperative MMSE, with no significant difference. After delirium, the mortality was higher than in the group without delirium. Figure 1 shows the Kaplan-Meier curve. Table 2 shows the proportion of patients with a loss of two points on the IADL scale and the mean NRS in the EQ5D according to whether or not postoperative delirium occurred.

The results of our univariate analysis are presented in Supplementary Materials. Among the risk factors for postoperative delirium, the median age of 70 years in the control group compared with 78 years in the delirium group ( $p<0.0001)$, MMSE (25 vs $26 ; p=0.003$ ), the left ventricular function $(55 \%$ vs $60 \% ; p=0.047)$ and the loss of hemoglobin in $\mathrm{g} / \mathrm{dL}(1.4 \mathrm{vs} 2.1 ; p=0.039)$ were noted. In addition, the duration of hospitalization was significantly longer in the delirium group in comparison to the control group (three days vs two days; $p=0.001$ ). The results of our multivariate analysis are presented in Table 3 . The risk factors for delirium were, age of more than 74 years (OR: 4.1; 95\%CI: $1.9-8.5 ; p=0.0001)$, and postoperative atrial fibrillation (OR: 2.6; 95\%CI: 1.16-5.99). Finally, preoperative MMSE, transfusion and chronic renal failure were not found to be a risk factor for delirium.

\section{Discussion}

In this work, we showed for the first time that POD was an independent risk factor of poor patient evolution at three years after cardiac surgery. In addition, the other independent risk factors were female gender, diabetes, and a Euroscore 2 more than 1.5. It has been demonstrated that more diabetic patients develop POD after cardiac surgery than nondiabetic patients. Elevated preoperative level of $\mathrm{HbA1c}$ was an independent risk factor for postcardiac surgery delirium 
Table 2 Quality of Life and Autonomy After POD at Three Years

\begin{tabular}{|l|l|l|l|l|}
\hline & Total $(\mathbf{n = 1 4 3 )}$ & No POD (n=99) & POD (n=44) & P (POD vs No POD) \\
\hline EQ5D NRS, mean (IQR) & $70(55-85)$ & $75(60-90)$ & $60(50-75)$ & 0.000 I \\
Loss of autonomy, n (\%) & $12.6(18)$ & $7(7 \%)$ & $11(25 \%)$ & 0.000 I \\
\hline
\end{tabular}

Notes: This table shows the \% of patients with lost of autonomy ( -2 points in the IADL scale) and the level of quality of life with the median response in the EQ5D NRS score. Results are also showed according to the presence or absence of delirium. EQ5D were compare with Mann-Whitney tests. Lost of autonomy was compared with chisquared test.

Abbreviations: POD, postoperative delirium; EQ5D, French version of EuroQuol 5D; IADL, instrumental activities of daily living.

Table 3 Risk Factors for Postoperative Delirium: Multivariate Analysis

\begin{tabular}{|l|l|l|l|l|}
\hline & P & OR & OR Lower Level (95\%) & OR Upper Level (95\%) \\
\hline MMSE D-I & 0.06 & 0.9 & 0.8 & 1.004 \\
Postoperative FA & 0.02 & 2.6 & 1.16 & 5.99 \\
Red Blood Cell Transfusion & 0.2 & 1.6 & 0.77 & 3.4 \\
Age $>$ 74 years & 0.0001 & 4.1 & 1.9 & 8.5 \\
Chronic renal failure & 0.1 & 1.97 & 0.89 & 4.4 \\
\hline
\end{tabular}

Notes: Occurrence of postoperative delirium was modeled as a function of all predictors that differed $(p<0.10)$ in the univariate analyses. Multivariate logistic regression analysis was performed by using a backward (conditional) stepwise procedure. AUC of this model was 0.78 , the Hosmer-Lemeshow test was 0.95 ; $73.4 \%$ of patients were high-ranked by this model.

regardless of the diagnosis of diabetes. ${ }^{6}$ In this cardiac surgery population, the presence of a single ischemic heart disease was protective. In addition, QOL scores were significantly lower after delirium, as was the case of the incidence of patients who had had a loss of autonomy. Mortality was higher in the group of patients with delirium. It was shown that mortality was associated with the occurrence of POD. ${ }^{14}$ In addition, it was previously demonstrated that over time, POD reduced the quality of life after surgery. ${ }^{12,15}$ After POD, the quality of life six and nine months postoperatively seemed particularly reduced due to the occurrence of problems related to anxiety/depression. However, in these works, the quality of life was only evaluated early between six months and one year postoperatively. ${ }^{12,15,16}$ In our work we show that this change persists over time, whilst the cognitive effects following delirium are known to regress. ${ }^{7,17}$ In addition, to our knowledge, there is no data in the literature that connects loss of autonomy to POD. POD could be considered as a fragility marker. Patients would in the future be more likely to develop problems leading to reduced quality of life and dependence. POD seems to actually impact patient outcome over time following surgery. Consequently, the occurrence is a complication that not only increases short-term mortality and morbidity during hospitalization, ${ }^{14}$ but also impacts patient outcome over the long-term and beyond the first year after surgery.

We also confirm the connection between the occurrence of POD and early alterations in cognitive performances. ${ }^{7,17}$
In fact, the patients with POD had a significant decrease in the MMSE seven days after surgery. While the MMSE is a rudimentary cognition evaluation tool, our results are similar to the studies that evaluated cognition with a battery of neuropsychological tests. The impact of delirium on cognitive performances seems to be transitory. After one year, cognitive changes induced by delirium were no significant. ${ }^{17}$ The fall in MMSE during hospitalization was not related to the occurrence in significant alterations in the primary endpoint. POCD present on discharge from hospital seems to be nondetermining of patient outcome in three years. POCD are described as regressive over time. ${ }^{7,9,18}$ Their impacts on the level of autonomy and the QOL can therefore also decline over time.

One limitation of our study could be that, unlike the other studies $^{12,16}$ in general we only included coronary bypass surgery, we also included patients who had undergone aortic and mitral valve replacement surgery, as well as surgeries in which valve replacements were associated with coronary bypass. Usually, patients who have undergone valvular surgeries have greater surgical risks than those who have undergone CABG. ${ }^{19}$ In our study, patients with only ischemic heart disease were also protected in terms of the occurrence of a poor evolution. Therefore, our population is similar to the population encountered in current practice, including patients at low operating risk and those at higher risk. There are several preoperative risk factors in the patients that could contribute to POD and POCD 
development significantly, such as, cerebrovascular incident, dementia, depression, neurodegenerative disease, poor cardiac function, prior cardiac or carotid surgery. Because such patients were not excluded from the research it could be a confusing factor. Finally, this was a singlecenter study with limited generalizability.

\section{Conclusion}

In this study, we showed that postoperative delirium impacted surgical follow-up by significantly altering patient outcome three years after heart surgery. Similarly, female gender, diabetes and Euroscore 2 influenced the outcome. The presence of ischemic heart disease was a protective factor.

Therefore, postoperative delirium is a postoperative complication that changes not only short-term, but also long-term patient outcome. In the future, strategies should be implemented to minimize the occurrence and to test whether these strategies reduce the incidence and improve patient outcome.

\section{Ethics}

Clinical trial number: local research ethics committee in 2015 (no. 78-1014).

\section{Funding}

Support was provided from CHU Toulouse, anesthesiology and ICU department and INSERM U1048.

\section{Disclosure}

The authors report no conflicts of interest in this work.

\section{References}

1. Evered L, Silbert B, Knopman DS, et al. Nomenclature Consensus Working Group a: recommendations for the nomenclature of cognitive change associated with anaesthesia and surgery-2018. Br J Anaesth. 2018;121(5):1005-1012. doi:10.1016/j.bja.2017.11.087

2. van Harten AE, Scheeren TWL, Absalom AR. A review of postoperative cognitive dysfunction and neuroinflammation associated with cardiac surgery and anaesthesia. Anaesthesia. 2012;67(3):280-293. doi:10.1111/j.1365-2044.2011.07008.x

3. Newman MF, Kirchner JL, Phillips-Bute B, et al. Neurological Outcome Research Group and the Cardiothoracic Anesthesiology Research Endeavors Investigators: longitudinal assessment of neurocognitive function after coronary-artery bypass surgery. $N$ Engl J Med. 2001;344(6):395-402. doi:10.1056/NEJM200102083440601
4. Knipp SC, Matatko N, Wilhelm H, et al. Cognitive outcomes three years after coronary artery bypass surgery: relation to diffusion-weighted magnetic resonance imaging. Ann Thorac Surg. 2008;85(3):872-879. doi:10.1016/j.athoracsur.2007.10.083

5. Marra A, Kotfis K, Hosie A, et al. Delirium monitoring: yes or no? That is the question. Am J Crit Care. 2019;28(2):127-135. doi:10.4037/ajec2019874

6. Kotfis K, Szylińska A, Listewnik M, Brykczyński M, Ely EW, Rotter I. Diabetes and elevated preoperative HbAlc level as risk factors for postoperative delirium after cardiac surgery: an observational cohort study. Neuropsychiatr Dis Treat. 2019;15:511-521. doi:10.2147/NDT.S196973

7. Saczynski JS, Marcantonio ER, Quach L, et al. Cognitive trajectories after postoperative delirium. $N$ Engl J Med. 2012;367(1):30-39. doi:10.1056/NEJMoa1112923

8. Sauër AC, Veldhuijzen DS, Ottens TH, Slooter AJC, Kalkman CJ, van Dijk D. Association between delirium and cognitive change after cardiac surgery. Br J Anaesth. 2017;119(2):308-315. doi:10.1093/bja/ aex053

9. Nadelson MR, Sanders RD, Avidan MS. Perioperative cognitive trajectory in adults. Br J Anaesth. 2014;112:440-451.

10. Kotfis K, Szylińska A, Listewnik M, et al. Early delirium after cardiac surgery: an analysis of incidence and risk factors in elderly ( $\geq 65$ years) and very elderly ( $\geq 80$ years) patients. Clin Interv Aging. 2018;13:1061-1070. doi:10.2147/CIA.S166909

11. Glumac S, Kardum G, Sodic L, Supe-Domic D, Karanovic N. Effects of dexamethasone on early cognitive decline after cardiac surgery: a randomised controlled trial. Eur J Anaesthesiol. 2017;34 (11):776-784. doi:10.1097/EJA.0000000000000647

12. Chen Y, Ding S, Tao X, et al. The quality of life of patients developed delirium after coronary artery bypass grafting is determined by cognitive function after discharge: a cross-sectional study. Int $J$ Nurs Pract. 2017;23.

13. Maniar HS, Lindman BR, Escallier K, et al. Delirium after surgical and transcatheter aortic valve replacement is associated with increased mortality. J Thorac Cardiovasc Surg. 2016;151(3):815823.e2. doi:10.1016/j.jtcvs.2015.10.114

14. Deiner S, Silverstein JH. Postoperative delirium and cognitive dysfunction. Br J Anaesth. 2009;103(Suppl 1):i41-6. doi:10.1093/ bja/aep291

15. Humphreys JM, Denson LA, Baker RA, Tully PJ. The importance of depression and alcohol use in coronary artery bypass graft surgery patients: risk factors for delirium and poorer quality of life. $J$ Geriatr Cardiol. 2016;13:51-57.

16. Koster S, Hensens AG, Schuurmans MJ, van der Palen J. Consequences of delirium after cardiac operations. Ann Thorac Surg. 2012;93(3):705-711. doi:10.1016/j.athoracsur.2011.07.006

17. Brown CH, Probert J, Healy R, et al. Cognitive decline after delirium in patients undergoing cardiac surgery. Anesthesiology. 2018;129 (3):406-416. doi:10.1097/ALN.0000000000002253

18. Moller JT, Cluitmans P, Rasmussen LS, et al. Long-term postoperative cognitive dysfunction in the elderly ISPOCD1 study. ISPOCD investigators. International Study of Post-Operative Cognitive Dysfunction. Lancet. 1998;351(9106):857-861. doi:10.1016/S01406736(97)07382-0

19. Velicki L, Cemerlic-Adjic N, Pavlovic K, et al. Clinical performance of the EuroSCORE II compared with the previous EuroSCORE iterations. Thorac Cardiovasc Surg. 2014;62(04):288-297. doi:10.1055/s-0034-1367734 


\section{Publish your work in this journal}

Clinical Interventions in Aging is an international, peer-reviewed journal focusing on evidence-based reports on the value or lack thereof of treatments intended to prevent or delay the onset of maladaptive correlates of aging in human beings. This journal is indexed on PubMed Central, MedLine, CAS, Scopus and the Elsevier
Bibliographic databases. The manuscript management system is completely online and includes a very quick and fair peer-review system, which is all easy to use. Visit http://www.dovepress.com/ testimonials.php to read real quotes from published authors.

Submit your manuscript here: https://www.dovepress.com/clinical-interventions-in-aging-journal 